\title{
Electrical properties of biodegradable poly(E-caprolactone):lithium thiocyanate complexed polymer electrolyte films
}

\author{
M. Ravi ${ }^{\mathrm{a}}$, Shenhua Song ${ }^{\mathrm{a}, *}$, Kunming Gu$^{\mathrm{b}}$, Jiaoning Tang ${ }^{\mathrm{b}}$, Zhongyi Zhang ${ }^{\mathrm{c}}$ \\ ${ }^{a}$ Shenzhen Key Laboratory of Advanced Materials, Department of Materials Science and \\ Engineering, Shenzhen Graduate School, Harbin Institute of Technology, Shenzhen 518055, \\ China. \\ ${ }^{\mathrm{b}}$ College of Materials Science and Engineering, Shenzhen University, Shenzhen 518060, China. \\ ${ }^{\mathrm{c}}$ Advanced Polymer and Composites (APC) Research Group, School of Engineering, University \\ of Portsmouth, Portsmouth, Hampshire PO1 3DJ, UK
}

\footnotetext{
${ }^{*}$ Corresponding author. Tel.: +86-755-26033465; fax: +86-755-26033504. E-mail address: shsong@hitsz.edu.cn (S.-H. Song).
} 


\begin{abstract}
:
Lithium ion conducting polymer electrolyte films based on biodegradable poly $(\varepsilon-$ caprolactone) (PCL) complexed with lithium thiocyanate (LiSCN) salt were prepared by solution cast technique. Thermal and electrical properties of the polymer electrolyte films were studied using differential scanning calorimetry (DSC) and AC impedance spectroscopy. In order to investigate the ion conduction mechanism and relaxation behavior of complex polymer electrolyte films, the conductivity, dielectric constant, loss tangent and electric modulus were analyzed as a function of frequency and temperature. The variation of conductivity with frequency obeyed the Johnscher's power law. The dielectric constant exhibited a higher value at a lower frequency and increased with rising temperature due to the polar nature of host polymer. The activation energies for both DC conductivity and relaxation had the same value $(\sim 0.87 \mathrm{eV})$, implying that the charge carriers responsible for both conduction and relaxation were the same.
\end{abstract}

Keywords: Polymer electrolyte, thermal analysis, Ion conduction mechanism, Impedance spectroscopy 


\section{Introduction}

Solvent-free ion conducting polymer-metal salt complexes belong to the division of superionic materials known as solid polymer electrolytes (SPEs). These materials have gained scientific and technological importance as electrolyte materials for the development of solid state electrochemical devices such as batteries, fuel cells, electrochromic windows and supercapacitors [1,2]. SPEs have several advantages over conventional liquid electrolytes, such as improved safety, ease preparation of thin films, leakage proof nature, low cost, light weight, good mechanical stability, high energy density and wide electrochemical stability windows. Therefore, a number of researchers focused on preparing good SPEs for solid state ionic device applications. However, many polymers involved create environmental problems, e.g., global warming due to their long degradable time. To mitigate these problems, several eco-friendly and biodegradable materials such as cellulose, starch, chitosan, poly (vinyl alcohol) and poly ( $\varepsilon$ caprolactone) are being used as a host polymer for the preparation of solid polymer electrolytes [3-8] Among these polymers, polyester-based biodegradable polymeric electrolytes are among the most promising polymer ionic conductors because their unique structures are able to facilitate fast ion transport. Poly( $\varepsilon$-caprolactone) (PCL) is a polyester-based biodegradable polymer and is nontoxic and widely used in different biomedical applications $[9,10]$. PCL possesses a melting point of approximately $60^{\circ} \mathrm{C}$ and its glass transition temperature is around $-60{ }^{\circ} \mathrm{C}$. It is derived from crude oil and prepared by ring opening polymerization of $\varepsilon$-caprolactone using a stannous octoate as catalyst. Due to its low glass transition temperature, the polymer chain exhibits segmental motion which helps transport of ions from one complexation site to another[11, 12]. PCL also possesses a carbonyl group and a Lewis base ester oxygen which can coordinate cations. A strong electron donating nature of this ester oxygen of carbonyl would tend to 
complex with cation, and thus it can be selected as a good polymer host for ionic device applications.

A large number of $\mathrm{Li}^{+}, \mathrm{Na}^{+}, \mathrm{K}^{+}, \mathrm{Mg}^{2+}$ conducting polymer electrolytes have been reported in literature [13]. In order to develop a well-complexed polymer-metal salt system, the selection of polymer as well as the metal salt plays a key role. To completely dissolve the salt into the polymer host, the lattice energy of the salt has to be compensated by the exothermic ionsolvation energy. This condition contributes to the reduction in the change of free energy involved in the solvation process. The choice of cation is restricted to a group of small ions because of the requirements for the electrolyte in the given application, and hence the size of the anion plays an important role in satisfying the criterion of lattice energy. From the anion-cation table, the comparison of the sizes of anions and their solubilities in PEO are well reviewed by Vincent et.al [14]. It is found that anions with larger radii possess less lattice energies and those with lower charge densities have a less tendency to form tight ion-pairs. Therefore, the most common anions facilitating complexation are $\mathrm{I}^{-}, \mathrm{ClO}_{4}{ }^{-}, \mathrm{CF}_{3} \mathrm{SO}_{3}{ }^{-}, \mathrm{SCN}^{-}, \mathrm{BF}_{4}{ }^{-}$and $\mathrm{AsF}_{6}{ }^{-}$. The coordination of the cations, the functional groups of the polymer and the stability of the complex observe the Hard Soft Acid Base (HSAB) principle $[13,15]$. The lithium thiocyanate (LiSCN) is a good dopant because it is thermally stable, nontoxic and insensitive to ambient moisture as compared to other lithium salts. Lithium can easily donates (loses) electrons to become a positive $\mathrm{Li}^{+}$which has a small ionic radius $(0.9 \AA)$ and the presence of thiocyanate in the lithium salt is a good anion of choice. As being aforementioned, the polyester-based PCL was selected as the host polymer and the lithium thiocyanate (LiSCN) as the dopant salt to fabricate polymer electrolyte films for lithium battery applications or electrochemical cell applications. 
Complex impedance spectroscopy (CIS) is a well-established method to study the electrical and dielectric properties of materials, which give four inversely related formalisms, such as impedance $Z^{*}$, electric modulus $\mathrm{M}^{*}$, admittance $\mathrm{Y}^{*}$ and permittivity $\varepsilon^{*}$. These complex formalisms indicate dielectric properties, such as dielectric constant, loss tangent, and electric modulus. The frequency and temperature-dependent dielectric properties of polymer electrolytes are very sensitive due to the motion of charge species and polymer dipoles, which reveals information on the ion conduction mechanism and its relationship with the dielectric parameters in the system. Several research studies are available on the electrical and dielectric properties of biodegradable polymer electrolytes and they reported the maximum conductivity values at room temperature, which are (i) $7.15 \times 10^{-7} \mathrm{~S} \mathrm{~cm}^{-1}$ for the $70 \mathrm{PVA}: 30 \mathrm{AgNO}_{3}$ [16], (ii) $2.2 \times 10^{-7} \mathrm{~S} \mathrm{~cm}^{-1}$ for the 56chitosan-44LiOAc [ 17], (iii) $2.10 \times 10^{-6} \mathrm{~S} \mathrm{~cm}^{-1}$ for the 75 methyl cellulose: $25 \mathrm{NH}_{4} \mathrm{NO}_{3}$ [18], (iv) $4.68 \times 10^{-5} \mathrm{~S} \mathrm{~cm}^{-1}$ for the $65 \mathrm{Starch}: 35 \mathrm{LiI}$ [19] and (v) $1.17 \times 10^{-6} \mathrm{~S} \mathrm{~cm}^{-1}$ for the 55(chitosan+PVA):45 $\mathrm{NH}_{4} \mathrm{I}[20]$. These conductivity values are comparable with those of some PCL:lithium salt complexed polymer electrolytes, which are (i) $1.1 \times 10^{-7} \mathrm{~S} \mathrm{~cm}^{-1}$ for the 88PCL: $12 \mathrm{LiBF}_{4}$, (ii) $1.26 \times 10^{-6} \mathrm{~S} \mathrm{~cm}^{-1}$ for the 90PCL:10 $\mathrm{LiClO}_{4}$ and (iii) $3.6 \times 10^{-5} \mathrm{~S} \mathrm{~cm}^{-1}$ for the 90PCL: $10 \mathrm{LiCF}_{3} \mathrm{SO}_{3}[21,22]$ at room temperature but these results are very few. So far there have been few detailed studies on the electrical and dielectric properties of PCL:lithium salt complexed polymer electrolytes. Hence, this study aims to explore the complete ion conduction mechanism by studying the electrical properties of the PCL:LiSCN complex polymer electrolyte systems.

\section{Experimental Details}

2.1 Materials 
The poly( $\varepsilon$-caprolactone) (PCL) with $\mathrm{Mn}=80 \mathrm{kDa}$ was procured from Sigma Aldrich and used as received. Lithium thiocyanate (LiSCN) was obtained from Sigma Aldrich and dried in an oven at $45^{\circ} \mathrm{C}$ for $24 \mathrm{~h}$ and stored in desiccators prior to use. Chloroform with a purity of $99 \%$ from Sigma Aldrich was used as the common solvent for the polymer film preparation.

\subsection{Polymer film preparation}

Pure poly(e-caprolactone) (PCL) and PCL:LiSCN complexed polymer electrolyte films were prepared using a standard solution cast technique. Initially, the PCL was added to chloroform in the predetermined quantity with stirring magnetically at room temperature for complete dissolution. 5, 10, 15 and $20 \mathrm{wt} \%$ of LiSCN salt were dissolved in chloroform and added to the PCL polymer solution under continuous stirring for $12 \mathrm{~h}$ to prepare homogeneous solution. Finally, viscous solutions were poured into glass petri dishes and thus the chloroform was allowed to evaporate slowly at ambient temperature to obtain free standing polymer electrolyte films at the bottom of the dishes. The films were dried at room temperature for6htoremoveanytracesoftheresidualsolvent in the films. The resulting polymer films were semitransparent and flexible in nature. The prepared films were stored in vacuum desiccators to avoid any moisture absorption prior to subsequent characterization. The thickness of these films was determined by the mechanical stylus method using a German made 'Perthometer' and was approximately $130 \mu \mathrm{m}$ with an accuracy of about $\pm 5 \mu \mathrm{m}$. The prepared films, as shown in Fig. 1, are self-standing and semi-transparent in nature.

\subsection{Characterization}

In order to understand the thermal history and stability of the present polymer electrolytes, differential scanning calorimetry (DSC) measurements were made on an STA 449F3 Jupiter thermal analyzer at a heating rate of $5^{\circ} \mathrm{C} \min ^{-1}$ under a nitrogen atmosphere in the 
temperature range of 30 to $150^{\circ} \mathrm{C}$. Surface morphology and microscopic feature of the polymer films were examined using optical microscopy. AC impedance spectroscopy is a well established method to study the electrical and dielectric properties of polymer films. In the present study, the impedance measurements were carried out using a computer controlled Solartron SI 1255 HF frequency response analyzer in conjunction with a Solartron 1296 dielectric interface. All the measurements were undertaken up to the melting temperature of the PCL $(333 \mathrm{~K})$ in the frequency range $0.1 \mathrm{~Hz}-1 \mathrm{MHz}$. The samples were dried in vacuum condition for $1 \mathrm{~h}$ and subsequently the measurements were recorded by sandwiching the electrolyte film between two stainless steel electrodes in a temperature controlled furnace. The electrochemical stability of the PCL:LiSCN complexed polymer electrolyte films was evaluated by cyclic voltammetry (CV) using an electrochemical analyzer (Model: CHI 760D, CH Instruments, China)

\section{Results and Discussion}

\subsection{Thermal analysis}

DSC measurements were carried out to determine the melting temperature $\left(T_{\mathrm{m}}\right)$ and relative percentage of crystallinity $\left(\chi_{c}\right)$ of polymer electrolytes. Fig. 2 shows the DSC curves of pure PCL and PCL:LiSCN complexes with different compositions in the temperature range of 30 to $95^{\circ} \mathrm{C}$. Clearly, the pure PCL exhibits a relatively sharp endothermic peak at $64{ }^{\circ} \mathrm{C}$, indicating the melting of pure PCL. This is attributed to the crystalline melting of PCL, which shows the presence of uncomplexed crystalline phase. The melting temperature $\left(T_{\mathrm{m}}\right)$ of pure PCL shifts to lower temperatures when the salt is added. The decrease of $T_{\mathrm{m}}$ upon the salt addition is not uncommon and is associated with the decrease in the size of spherulites [23] and their surface free energies. When the salt concentration is further increased, $T_{\mathrm{m}}$ is further lowered due to the suppression of crystallites, thereby increasing the amorphous content in the polymer matrix. As a 
result of additional amorphous surroundings being trapped in or adjacent to the crystalline matrix, the suppressed crystalline part of the PCL complex would melt at a lower temperature [21].

The relative crystallinity of complexed polymer electrolytes $\left(\chi_{\mathrm{c}}\right)$ can be calculated from their DSC curves based on the following equation with the assumption that the pure PCL polymer is $100 \%$ crystalline $[24,25]$.

$$
\chi_{\mathrm{c}}(\%)=\left[\Delta H_{\mathrm{m}} / \Delta H_{\mathrm{m}}^{0}\right] \times 100
$$

where $\Delta H_{\mathrm{m}}^{0}$ is the crystalline melting enthalpy of the pure PCL, and $\Delta H_{\mathrm{m}}$ is the melting enthalpy of the PCL:LiSCN complexed polymer film. The intensity of melting endothermic event decreases and shifts to lower temperatures with increasing salt concentration in the complexed samples. This appears to be more apparent with a decrease in the enthalpy of melting $\left(\Delta H_{\mathrm{m}}\right)$, indicating a reduction in the degree of crystallinity of the polymer electrolyte films with increasing salt concentration, and thus leading to an increase in the amorphous phase. The measured values of melting temperature $\left(T_{\mathrm{m}}\right)$, melting enthalpy $\left(\Delta H_{\mathrm{m})}\right.$ and relative crystallinity $\left(\chi_{c}\right)$ are listed in Table 1.

The reduction in melting temperature and crystalline fraction should be attributed to the inhibition of crystallization by the dissolved ions. Since more salt is accommodated in PCL, it suppresses the crystalline phase in the polymer. The polymeric chain in the amorphous phase is more flexible and mobile, which results in an enhancement of segmental motion of the polymer $[26,27]$. The low value of $\chi_{\mathrm{c}}$ demonstrates the high amorphous nature of the aforementioned polymer electrolyte. When the LiSCN salt concentration exceeds $15 \mathrm{wt} \%$, the values of $\chi_{\mathrm{c}}, \Delta H_{\mathrm{m}}$ and $T_{\mathrm{m}}$ are all increased. This may be due to the reorganization of the polymer matrix. 


\subsection{Surface analysis}

Fig. 3 shows the optical micrographs of pure PCL film and PCL: LiSCN complexes with different LiSCN concentrations. Typical spherulitic texture along with dark boundaries can be seen in the pure PCL film, demonstrating its semi-crystalline nature. This type of surface morphology was also observed for other semi-crystalline polymers, such as (PEO) $6 \mathrm{NaPO}_{3}$ and PEO: $\mathrm{Mg}\left(\mathrm{CF}_{3} \mathrm{SO}_{3}\right)_{2}$ polymer electrolytes $[28,29]$. The spherulitic texture in the polymer film shows its lamellar crystalline nature and the dark boundaries indicate the amorphous content in the polymer (Fig. 3a). With the addition of LiSCN salt in the PCL polymer matrix, there are significant surface morphology changes (see Figs. $3 b$ and 3c). It is seen that the dark boundary regions increase with increasing LiSCN content up to $15 \mathrm{wt} \%$, indicating an increase in the amorphous content in the polymer-salt complexes. In addition, the surface of spherulites becomes smoother with increasing salt concentration, showing an increase in intra-spherulitic amorphous phase. This reduction in crystallinity has also been confirmed above by the DSC results. When the salt concentration exceeds $15 \mathrm{wt} \%$, the dark boundary regions are reduced along with the increment in lamellae texture. This may be due to the reorganization of the polymer matrix (see Fig. 3d).

\subsection{Electrical conductivity analysis}

The frequency dependence of electrical response is a common response of solid polymer electrolytes and it is a versatile approach to understanding the ion conduction mechanism [30]. Fig. 4 shows the frequency dependences of ionic conductivity of the PCL:LiSCN (95:05) complexed polymer electrolyte film at different temperatures. Clearly, two distinct regions are observed in the measured frequency range, namely, low frequency plateau region and high 
frequency dispersion region. The low frequency plateau region describes the space charge polarization at the electrode-electrolyte interface and is associated with the DC conductivity $\left(\sigma_{\mathrm{dc}}\right)$ of the polymer electrolyte. At higher frequencies the forward and backward ion displacements take place simultaneously, leading to an increase in ionic conductivity with increasing frequency. This is related to the AC conductivity $\left(\sigma_{\mathrm{ac}}\right)$. However, at low frequencies the conductivity increases with increasing temperature but at high frequencies the conductivity variation with temperature is nearly the same.

The total conductivity $\sigma(\omega)$ can be expressed as the sum of dc and ac components according to the Johnscher's universal power law equation [31]

$$
\sigma(\omega)=\sigma_{\mathrm{dc}}+\sigma_{\mathrm{ac}}
$$

where $\sigma_{\mathrm{dc}}$ is the frequency-independent conductivity and $\sigma_{\mathrm{ac}}$ is the frequency-dependent conductivity. $\sigma_{\mathrm{ac}}$ is given by $A \omega^{n}$, where $A$ is a frequency-independent temperature-related parameter, $\omega$ is the angular frequency given by $2 \pi f$, and $n$ is the temperature-dependent frequency exponent. This power law behavior is a universal property of materials, which is related to the dynamics of ion hopping conduction [32], and has widely been observed in conductive polymers [33]. According to the jump relaxation model [34], the exponent $n$ can be expressed as

$$
n=\left[b_{\mathrm{r}} / s_{\mathrm{r}}\right]
$$

where $b_{\mathrm{r}}$ is the back hop rate and $s_{\mathrm{r}}$ is the site relaxation rate. The Coulomb repulsive interaction among mobile ions induces the back hop which is the backward motion of a hopping ion to its initial site. The site relaxation is the shift of a site potential minimum to the position of the hopping ion due to the rearrangement of neighboring ions [35]. If $n<1$, the backward hopping is slower than the site relaxation. This results in a translational motion of the $\mathrm{Li}^{+}$ion. However, if 
$n>1$, the backward hopping is faster than the site relaxation. Using the conductivity spectra of the PCL:LiSCN (95:05) polymer electrolyte (Fig. 4), the evaluated values of exponent $n$ are almost the same at all the temperatures, being approximately 1.20. The value of $n$ greater than one is either due to the presence of a bad site for the next hop, or the Columbic repulsion between the mobile ions.

Currently, a number of theoretical models, such as small polaron (SP), overlapping large polaron-tunneling (OLPT), correlated barrier-hopping $(\mathrm{CBH})$ and quantum mechanical tunneling (QMT) models, have been proposed to correlate the temperature dependence of exponent $n$ with the conduction mechanism of the material. As far as these models are concerned, the value of $n$ in the Johnscher's universal power law is evaluated at each individual temperature. In the SP model, $n$ increases with increasing temperature. In the OLPT model, $n$ decreases to a minimum value with increasing temperature and increases again as the temperature further increases. In the CBM model, $n$ decreases with increasing temperature, but no minimum is observed. In the QMT model, $n$ is independent of temperature. From the behavior of exponent $n$ with temperature in the PCL:LiSCN (95:05) polymer electrolyte system (Fig. 4), it can be inferred that the QMT model is more applicable to explain the conduction mechanism.

As can be seen in Fig. 4, the frequency at which the dispersion becomes distinguished shifts to higher frequencies with increasing temperature. In other words, the bulk relaxation shifts to higher frequencies with increasing temperature, resulting in an increase in dc conductivity. In the full temperature range, the de conductivity values of PCL:LiSCN polymer electrolytes were obtained from the intercept of the plateau region on the conductivity axis (yaxis) at zero frequency. As an inset in Fig. 4, the evaluated DC conductivity of the PCL:LiSCN (95:05) polymer electrolyte $(\sigma)$ is plotted as a function of temperature (T). It is evident that $\ln$ 
$(\sigma T)$ increases linearly with increasing reciprocal temperature $(1 / T)$. This means that the conductivity follows an Arrhenius equation $\sigma T=\sigma_{0} \exp \left(-E_{\mathrm{a}} / k T\right)$, where $E_{\mathrm{a}}$ is the activation energy for conduction, $\sigma_{0}$ is the pre-exponential constant, $k$ is the Boltzmann constant and $T$ is the absolute temperature. The activation energy obtained by linear fitting is $0.87 \mathrm{eV}$. The increase of conductivity with increasing temperature may be due to the decrease of viscosity and hence the increase of chain flexibility [36].

Fig. 5 shows the frequency-dependent ionic conductivities of PCL:LiSCN complexed polymer electrolyte films with different LiSCN salt contents. It is evident that the electrical conductivity increases with increasing LiSCN content until $15 \mathrm{wt} \%$ and then decreases with further increasing LiSCN content. The conductivity increase with LiSCN content may be attributed to the increase in the number of mobile charge carriers and also to the decrease in the crystallinity of the polymer, which reduces the energy barrier through fast ion transport. The reduction in the crystallinity of PCL:LiSCN complexed polymer electrolytes can also be seen from the DSC analysis. A polymer chain in the amorphous phase is more flexible, which results in an increase in the segmental motion of the polymer, thereby enhancing the ionic mobility [26]. The values of exponent $n$ evaluated with the conductivity spectra of the polymer electrolytes, as shown in Table 2, are varying from 1.20 to 0.96 in different LiSCN concentrations. The value of $n$ is less than one and has a minimum value of 0.96 for the $15 \mathrm{wt} \%$ LiSCN-doped sample, which could be due to the formation of free sites for $\mathrm{Li}^{+}$ion transport, i.e., the ions have a good site for their next hop and the backward motion is slower due to less Columbic interaction between the ions [37]. In the case of the salt concentration exceeding $15 \mathrm{wt} \%$, the number of free $\mathrm{Li}^{+}$ions may decrease because of the formation of ion pairs $\left(\mathrm{Li}^{+}---\mathrm{SCN}^{-}\right)$and ion triplets $\left(\mathrm{Li}^{+}\right.$--- $\mathrm{SCN}^{-}---$ $\left.\mathrm{Li}^{+}\right)$in the polymer matrix. These ion pairs and ion triplets hinder the ion transport and block the 
segmental motion of the polymer chains. Consequently, the overall conductivity is decreased.

The detailed values of conductivity are listed in Table 2 . It can be seen that the maximum ionic conductivity at room temperature, $0.104 \times 10^{-5} \mathrm{~S} \mathrm{~cm}^{-1}$, is well comparable with those available in the literature. It is worth mentioning that the electrical conductivity could be higher because the effective electrode area could be smaller than the apparent electrode area employed in the calculation as the sample-electrode contact is usually not $100 \%$. In the future research, it could be possible to increase the ionic conductivity value up to $\sim 10^{-3} \mathrm{~S} \mathrm{~cm}^{-1}$ by the addition of plasticizers (ethylene carbonate, propylene carbonate, etc), ionic liquids (1-butyl-3methylimidazolium hexafluorophosphate (BMIM- $\left.\mathrm{PF}_{6}\right)$, 1-butyl-3-methylimidazolium bis(trifluoromethylsulfonyl)imide (BMIMTFSI), 1-butyl-3-methylimidazolium tetrafluoroborate (BMIM-BF 4 ), 1-ethyl-3-methylimidazolium trifluoromethanesulfonate (EMITF), etc), and nanofillers $\left(\mathrm{TiO}_{2}, \mathrm{Al}_{2} \mathrm{O}_{3}, \mathrm{ZnO}\right.$, etc) in the $15 \mathrm{wt} \% \mathrm{LiSCN}$-complexed polymer electrolyte system. This magnitude of conductivity will be quite enough for practical ionic device applications [38$40]$.

3.4 Dielectric analysis

\subsubsection{Dielectric constant analysis}

The relative permittivity of polymer electrolyte films helps to understand the polarization effect at the electrode-electrolyte interface. The relative permittivity $\left(\varepsilon_{\mathrm{r}}^{*}\right)$ of a system is a dimensionless quantity and it is defined as the ratio of material permittivity $(\varepsilon)$, to the free-space permittivity $\left(\varepsilon_{0}\right)$. It can also be described in complex form (as a function of angular frequency) with real and imaginary components and these two components are $90^{\circ}$ out of phase.

$$
\varepsilon_{\mathrm{r}}^{*}(\omega)=\varepsilon(\omega) / \varepsilon_{0}=\varepsilon^{\prime}(\omega)-\mathrm{j} \varepsilon^{\prime \prime}(\omega)
$$


where $\varepsilon^{\prime}$ is the real part of complex permittivity, $\varepsilon^{\prime \prime}$ is the imaginary part of complex permittivity and $j=\sqrt{-1}$. Both the real and imaginary parts are of particular significance in ion conducting polymers. The real part of complex dielectric permittivity $\left(\varepsilon^{\prime}\right)$ has the same meaning as the ordinary dielectric constant of the material. It measures the energy stored in the material during each cycle, to be returned to the electric field at the end of the cycle, and it also represents the amount of dipole alignment in a given volume. The imaginary part of complex dielectric permittivity $\left(\varepsilon^{\prime \prime}\right)$ represents the energy loss of ion motion and dipole alignment when the polarity of electric field reverses rapidly, and it is related to the electrical conductivity of the materials. Fig. 6 shows the frequency variation of the dielectric constant ( $\left.\varepsilon^{\prime}\right)$ for the PCL:LiSCN $(90: 10)$ complexed polymer electrolyte film at different temperatures. A dispersion with high $\varepsilon^{\prime}$ values are observed in the low frequency range and is attributed to dielectric polarization. In the low frequency range, the ions are capable of moving in the direction of the electric field, but they are unable to be transported to the external circuit because of the blocking electrodes. This indicates that the electrode polarization and space charge effects are predominant. As far as all polymer electrolyte systems are concerned, two sources of dipoles are available within the frequency study. Mobile salt ions that dissociate into cation-anion pairs and localized molecular polar groups exhibit an imbalance of charge. Under the influence of an electric field, the ions tend to diffuse and migrate along the field. However, they are unable to cross the electrode-electrolyte interface because the blocking electrodes do not allow charge transfer in the external circuit. These limited and reversible trapped ions then accumulate at the electrode-electrolyte interface, becoming localized and forming a hetero charge layer. With the assumption that the thickness of the sample is much greater than this hetero charge layer, the charge density would increase rapidly, leading to electrode or interfacial polarization [41]. Another source of dipoles comes 
from the polar groups (carbonyl group) of the polymer chain. These carbonyl group permanent dipoles might also induce different dipoles surrounding them in an attempt to align favorably to the electric field through conformational changes. They rotate slightly from the equilibrium positions where positive charged regions displace towards the field and negative charged regions rotate away from the field, leading to the polarization effect [42]. These factors lead to a high dielectric constant value $\left(\sim 10^{5}\right)$ at a low frequency. It is seen that the dielectric constant decreases rapidly in the frequency range $0.1-10 \mathrm{kHz}$ and reaches a constant value at higher frequencies $(\mathrm{MHz})$. At very high frequencies, the periodic reversal of the field takes place so quickly that the charge carriers can hardly have time to orient themselves in the field direction, resulting in the observed frequency-independent $\varepsilon^{\prime}$ value [43].

As shown in Fig. 6, the dielectric constant increases with increasing temperature for the PCL:LiSCN (90:10) polymer electrolyte system. The variation of dielectric constant with temperature is different between non-polar and polar polymers. In general, $\varepsilon^{\prime}$ is independent of temperature for non-polar polymers. However, $\varepsilon^{\prime}$ increases with increasing temperature in the case of strong polar polymers. From this study, the behavior of $\varepsilon^{\prime}$ for PCL:LiSCN electrolytes is typically polar dielectric. When the temperature is increased, the low-frequency dispersion of dielectric constant shifts towards higher frequencies. At high frequencies, $\varepsilon^{\prime}$ of the polymer electrolyte is nearly constant with respect to the variation of temperature, which suggests the effective participation of dipoles in dielectric polarization of PCL. The observed dielectric constant behavior with frequency and temperature variations confirms that the electrical charge hopping mechanism governs both the charge transport and relaxation [44].

Fig. 7 shows the frequency dependence of dielectric constant $\left(\varepsilon^{\prime}\right)$ for PCL:LiSCN complexed polymer electrolyte films with different LiSCN concentrations. It can be observed 
that $\varepsilon^{\prime}$ increases with increasing LiSCN content up to a maximum value at $15 \mathrm{wt} \%$ and thereafter it decreases with further increasing salt content. A similar behavior is also observed in Fig. 5, i.e., the LiSCN-dependent conductivity and dielectric constant follows the same trend. The increase in $\varepsilon^{\prime}$ with increasing salt concentration is due to the increased number density of charge carriers as a result of salt dissociation in the polymer matrix. At a higher salt concentration ( $>15 \mathrm{wt} \%)$, the distance between ions become smaller and thus the Coulomb attraction increases, leading to ion re-association and formation of neutral ion pairs or large clusters [45]. This directly decreases the number density of charge carriers and their mobility with a reduced contribution to dielectric constant.

\subsubsection{Loss tangent $(\tan \delta)$ analysis}

The dielectric relaxation parameter of the polymer electrolytes can be obtained from the study of loss tangent $(\tan \delta)$ as a function of frequency. The loss tangent $(\tan \delta)$ is the ratio of loss factor $\left(\varepsilon^{\prime \prime}\right)$ to dielectric constant $\left(\varepsilon^{\prime}\right)$. It is a measure of the ratio of the electrical energy lost to the energy stored in a periodic field. It is given by

$$
\tan \delta=\varepsilon^{\prime \prime} / \varepsilon^{\prime}
$$

The frequency dependences of loss tangent for the PCL:LiSCN (95:05) complexed polymer electrolyte at different temperatures are represented in Fig. 8. It is clear that $\tan \delta$ increases with increasing frequency to reach a maximum value and thereafter decreases with further increasing frequency, making well defined peaks readily observed. At lower frequencies, $\tan \delta$ increases with increasing frequency as a result of the active element (ohmic) which is more dominant than the reactive element (capacitive). At higher frequencies $(\sim \mathrm{MHz}), \tan \delta$ decreases with increasing frequency because the reactive element grows in proportion to the frequency and also the ohmic element is independent of frequency [46]. The observed well-defined peak 
separates the electrode polarization and bulk relaxation processes of the material. The electrode polarization effect is observed in the low frequency range, whereas the bulk relaxation process occurs in the high frequency range [47]. At a low frequency, the mobile charge carriers accumulate on the electrode surface due to the slow periodic reversal of the applied electric field and the electrical double layer formation as a blocking layer. This layer would hinder the motion of $\mathrm{Li}^{+}$cations in the polymer network, which is a non-Debye nature of the polymer electrolyte [48].

It is also seen from Fig. 8 that the maximum $\tan \delta$ shifts to higher frequencies with increasing temperature, suggesting a dielectric relaxation process. This is a typical feature of polar dielectrics [49]. The natural logarithm of the frequency corresponding to the maximum of $\tan \delta\left(\ln f_{\max }\right)$ is plotted in Fig. 9 as a function of reciprocal temperature. It is a straight line and obeys the equation below

$$
f_{\max }=f_{0} \exp \left(-E_{\mathrm{m}} / k T\right)
$$

where $E_{\mathrm{m}}$ is the ion migration energy, $f_{0}$ is a constant, $k$ is the Boltzmann constant, and $T$ is the absolute temperature. The linear fitting can obtain $E_{\mathrm{m}}$ as $0.88 \mathrm{eV}$, which is in good agreement with the DC conductivity activation energy $E_{\mathrm{a}}(0.87 \mathrm{eV})$. This result suggests that the charge carriers responsible for both conductivity and relaxation are the same.

Fig. 10 shows the frequency dependences of normalized loss $\operatorname{tangent}\left[\tan \delta /(\tan \delta)_{\max }\right]$ for PCL:LiSCN complexes with different LiSCN concentrations. It is seen that all the PCL:LiSCN complexes show well defined peaks and the peak maximum shifts to higher frequencies with increasing salt concentration up to $15 \mathrm{wt} \%$. By taking Debye equation in an ideal case, the relaxation time $(\tau)$ can be evaluated by [46]

$$
2 \pi f_{\max } \tau=1
$$


The relaxation time values are calculated for all the PCL:LiSCN complexed polymer electrolytes at $303 \mathrm{~K}$ and are tabulated in Table 2. As seen, the shortest relaxation time is obtained for the 15 wt\% salt-doped polymer electrolyte, which is consistent with the conductivity analysis (Fig. 5). The amorphous region expands and the molecular packing becomes loose and weak at a high salt incorporation so that the polymer chain is more flexible to orient, resulting in a reduction in relaxation time.

\subsubsection{Electric modulus analysis}

The detailed dielectric behavior of polymer electrolytes can be satisfactorily explained by dielectric modulus analysis. The complex electric modulus has been used to investigate the conductivity relaxation phenomena; it suppresses the electrode polarization effects and gives a clearer picture of electrical properties inherited in the polymer electrolyte. The complex electric modulus can be calculated from the impedance data using the following equation

$$
M^{*}=\left(\varepsilon^{*}\right)^{-1}=M^{\prime}+\mathrm{j} M^{\prime \prime}=\frac{\varepsilon^{\prime}}{\varepsilon^{\prime 2}+\varepsilon^{\prime 2}}+\mathrm{j} \frac{\varepsilon^{\prime \prime}}{\varepsilon^{\prime 2}+\varepsilon^{\prime \prime 2}}
$$

where $M^{\prime}$ and $M^{\prime \prime}$ are the real and imaginary parts of the electric modulus and $\varepsilon^{\prime}$ and $\varepsilon^{\prime \prime}$ are the real and imaginary parts of the dielectric constant, respectively.

Fig. 11 shows the variation of $M^{\prime \prime}$ with frequency at different temperatures for the PCL:LiSCN (85:15) complex polymer electrolyte. It can be seen that the $M^{\prime \prime}$ value is very low (approaching zero) in the low frequency region at all the temperatures. This indicates that the electrode polarization phenomenon is negligible in this formulism [50]. The well-defined asymmetric peaks are observed at higher frequencies and the full width half maximums (FWHMs) of these peaks are broader than that of the ideal Debye peak, which is usually 
attributed to the deviation from Debye nature of the samples [51]. The peak height decreases with increasing temperature, suggesting a plurality relaxation mechanism [52].

The low frequency side of the peak $\left\{f<f_{\max }\right\}$ represents the range of frequencies at which the charge carriers are mobile and the ions can move over a long distance, i.e., the ions can successfully hop from one site to a neighboring site. The high frequency side of $M^{\prime \prime}$ peak $\left(f>f_{\max }\right)$ represents the range of frequencies at which the ions are spatially confined to their potential wells and they can just make localized motion within the well [53].The frequency corresponding to the peak of $M^{\prime \prime}$ is indicative of the transition from long-range to short-range motion with increasing frequency. The capacitance values were calculated at all the peak frequencies from the equation $M^{\prime \prime}=\varepsilon_{0} / 2 \mathrm{C}$ and they are in the order of magnitude of $\mu \mathrm{F}$, indicating that the observed peaks are attributed to the bulk relaxation effects of the material. The variation of normalized modulus $\left(M^{\prime \prime} / M^{\prime \prime}{ }_{\max }\right)$ with normalized frequency $\left(\log \left(f / f_{\max }\right)\right)$ for the PCL:LiSCN $(85: 15)$ complex polymer electrolyte at different temperatures is shown in Fig.12. It is also referred to as "master curve" and represents the scaling behavior of the sample. This scaling behavior of electric modulus curve helps us understand the temperature dependence of the dielectric process occurring in the material. The near perfect overlap of the modulus curves for all the temperatures indicates that the dynamical process (relaxation) occurring at different frequencies is independent of temperatures [54].The non-symmetric nature of the curves is well described by the Kohlrausch-Williums-Watts (KWW) function $[\phi(t)]$. This function represents the distribution of relaxation time in ion-conducting materials.

$$
\phi(t)=\exp \left(-t / \tau_{\sigma}\right)^{\beta}
$$

where $\tau_{\sigma}\left(\tau_{\sigma}=1 / 2 \pi f_{\mathrm{p}}\right)$ is the conductivity relaxation time and $\beta$ is the Kohlrausch exponent. The parameter $\beta$ describes the breadth of the distribution in the limits of $0 \leq \beta \leq 1$. The smaller the value 
of $\beta$ the larger the deviation of relaxation from Debye type relaxation $(\beta=1)$ [55]. The values of $\beta$ corresponding to all the temperatures for the PCL:LiSCN (85:15) complex polymer electrolyte have been calculated by using the formula $\beta=1.14 /$ FWHM, and they are almost the same $(0.736)$. A similar type of spectra has also been observed for the other PCL:LiSCN complexed polymer electrolytes, but their FWHMs values are different. The obtained $\beta$ values are listed in Table 2 for all the prepared PCL:LiSCN complex polymer electrolytes. Clearly, the value of $\beta$ is less than 1, indicating the non-Debye type relaxation nature of the material. This corresponds to slow polarization. The increase in the value of $\beta$ with increasing salt concentration is due to the increase in charge-carrier concentration [56]. This is in good agreement with the findings from conductance and dielectric analyses of the samples.

Recently, Woo et al. [24, 57] prepared the PCL-based proton conducting polymer electrolyte films and studied their structural, thermal and electrical properties by virtue of FTIR, DSC and impedance spectroscopy. The DSC results revealed that the melting temperature $\left(T_{\mathrm{m}}\right)$ and relative crystallinity $\left(\chi_{\mathrm{c}}\right)$ decreased with increasing $\mathrm{NH}_{4} \mathrm{SCN}$ salt content up to $26 \mathrm{wt} \%$ and then $T_{\mathrm{m}}$ and $\chi_{\mathrm{c}}$ both increased with further increasing $\mathrm{NH}_{4} \mathrm{SCN}$ salt concentration. For the 74PCL:26NH $\mathrm{NHCN}_{4} \mathrm{Scomplexed} \mathrm{polymer} \mathrm{electrolyte,} \mathrm{the} \mathrm{minimum} T_{\mathrm{m}}$ and $\chi_{\mathrm{c}}$ were obtained as $54.70{ }^{\circ} \mathrm{C}$ and $81 \%$. At room temperature, the electrical conductivity increased with increasing salt concentration and the maximum electrical conductivity of $4.6 \times 10^{-8} \mathrm{~S} \mathrm{~cm}^{-1}$ was obtained for the $74 \mathrm{PCL}: 26 \mathrm{NH}_{4} \mathrm{SCN}$ system. It was also reported that the variation of dielectric constant with salt concentration followed the same trend as the room-temperature conductivity. The welldefined peak was observed in loss tangent analysis and it shifted to higher frequencies with increasing salt concentration. The electrical modulus analysis revealed the non-Debye nature of 
the PCL: $\mathrm{NH}_{4} \mathrm{SCN}$ polymer electrolyte. Obviously, the present results in melting temperature, relative crystallinity and conductivity are well comparable with theirs.

Finally, it is worth mentioning that the electrochemical stability, i.e., the working voltage range of the electrolytes is an important parameter from their application in electrochemical devices such as batteries and super capacitors. The working voltage range of the 85PCL:15LiSCN complexed polymer electrolyte film was evaluated using cyclic voltammetry $(\mathrm{CV})$ in the two-electrode configurations with the stainless steel electrodes (electrode crosssection area $=0.853 \mathrm{~cm}^{-2}$ ). Fig. 13 shows the $\mathrm{CV}$ pattern of the electrolyte film recorded at a scan rate of $1 \mathrm{mV} \mathrm{s}^{-1}$. As can be seen, the current remains in a steady value in the voltage range of approximately -1.3 to $1.7 \mathrm{~V}$. This means that the potential window is in the range of -1.3 to $1.7 \mathrm{~V}(\sim 3 \mathrm{~V})$, which could be an acceptable working voltage for the device applications [58-60].

\section{Conclusions}

Environment-friendly lithium ion conducting PCL:LiSCN complex polymer electrolyte films have been prepared. The relative degree of crystallinity of PCL decreases with an increase of LiSCN concentration and the minimum relative degree of crystallinity is obtained for the 15wt\% LiSCN-doped film, being $~ 73.4 \%$. The conduction mechanism of PCL:LiSCN complexed polymer electrolytes follows the quantum mechanical tunneling model. The increase of ionic conductivity and dielectric constant with an increase in salt concentration up to $15 \mathrm{wt} \%$ indicates an increase of dissociated ions in the polymer matrix. The large dielectric constant value of the films appears at low frequencies and it increases with rising temperature, showing the polar nature of the material. The activation energy for DC conductivity and the loss tangent 
demonstrate that the charge carriers responsible for both conduction and relaxation are the same.

Outcomes from the modulus analysis confirm the non-Debye nature of the polymer electrolyte.

Acknowledgments: This research was supported by the Science and Technology Foundation of Shenzhen, China. 


\section{References}

[1] J.R. Mac Callum, C.A. Vincent, (Eds.), Polymer Electrolytes Reviews, Elsevier, Amsterdam, 1987.

[2] R.C. Agrawal, G.P. Pandey, Solid polymer electrolytes:materials designing and all-solidstate battery applications: an overview, J. Phys. D Appl. Phys. 41 (2008) 2223001-2230018.

[3] A.S. Samsudin, H.M. Lai, M.I.N. Isa, Biopolymer Materials Based Carboxymethyl Cellulose as a Proton Conducting Biopolymer Electrolyte for Application in Rechargeable Proton Battery, Electrochimica Acta 129 (2014) 1-13.

[4] M.F. Shukur, R. Ithnin, M.F.Z. Kdir, Electrical characterization of corn starch-LiOAc electrolytes and application in electrochemical double layer capacitor, Electrochimica Acta 136 (2014) 204-216.

[5] S. Rmesh, Chiam-Wen Liew, A. K. Arof, Ion conducting corn starch biopolymer electrolytes doped with ionic liquid 1-butyl-3-methylimidazoliu m hexafluorophosphate, J. Non-Cryst. Solids 357 (2011) 3654-3660.

[6] L.S. Ng, A.A. Mohamad, Effect of temperature on the performance of proton batteries based on chitosan - $\mathrm{NH}_{4} \mathrm{NO}_{3}$-EC membrane, J. Memb. Sci. 325 (2008) 653-657.

[7] I.S. Noor, S.R. Majid, A.K. Arof, Poly(vinyl alcohol)-LiBOB complexes for lithium -air cells, Electrochimica Acta 102 (2013) 149-160,

[8] C. Polo Fonseca, S. Neves, Electrochemical properties of a biodegradable polymer electrolyte applied to a rechargeable lithium battery, J. Power Sources 159 (2006) 712-716.

[9] A.G.A. Coombes, S.C. Rizzi, M. Williamson, J.E. Barralet, S. Downes, W.A. Wallace, Precipitation casting of polycaprolactone for applications in tissue engineering and drug Delivery, Biomaterials 25 (2004) 315-325. 
[10] S.S. Ray, M. Bousmina, Biodegradable polymers and their layered silicate

nanocomposites: In greening the 21 st century materials world,

Prog. Mater. Sci. 50 (2005) 962-1079.

[11] Roy L Abee, Martin Van Duin, Han Goossens, Crystallization Kinetics and Crystalline Morphology of Poly(e-caprolactone) in Blends with Grafted Rubber Particles, J. Polym. Sci. Part B: Polym. Phys. 48 (2010) 1438-1448.

[12] A.M. Christie, S.J. Lilley, E. Staunton, Y.G. Andreev, P.G. Bruce, Increasing the conductivity of crystalline polymer electrolytes, Nature 433 (2005) 50-53.

[13] P.G. Bruce, Solid State Electrochemistry, Cambridge University Press, Cambridge, 1995. [14] C.A. Vincent, Polymer electrolytes, Prog. Solid State Chem. 17(3) (1987) 145-261.

[15] F.M. Gra, Polymer electrolytes: Fundamentals and Technological Applications, VCH pulblications, New York, 1991

[16] G. Hirankumar, S. Selvasekarapandian, M.S. Bhuvaneswari, R. Baskaran, M. Vijayakumar, $\mathrm{Ag}^{+}$ion transport studies in a poly vinyl alcohol-based polymer electrolyte system, J. Solid State Electrochem. 10 (2006) 193-197.

[17] M.Z.A. Yahya, A.K. Arof, Conductivity and X-ray photoelectron studies on lithium acetate doped chitosan films, Carbohydrate polymers 55 (2004) 95-100.

[18] N.E.A. Shuhaimi, L.P. Teo, S.R. Majid, A.K. Arof, Transport studies of $\mathrm{NH}_{4} \mathrm{NO}_{3}$ doped methyl cellulose electrolyte, Synth. Met. 160 (2010) 1040-1044.

[19] M.H. Khanmirzaei, S. Ramesh, Studies on biodegradable polymer electrolyte rice starch (RS) complexed with lithium iodide. Ionics 20 (2014) 691-695.

[20] M.H. Buraidah, A.K. Arof, characterization of Chitosan/PVA blended electrolyte doped with $\mathrm{NH}_{4} \mathrm{I}$, J. Non-Crys. Solids 357 (2011) 3261-3266. 
[21] C.P. Fonseca, D.S. Rosa, F. Gaboardi, S. Neves, Development of a biodegradable polymer electrolyte for rechargeable batteries, J. Power Sources 155 (2006) 381-384.

[22] C.P. Fonseca, F.Jr. Cavalcante, F.A. Amaral, C.A. Zani Souza, S. Neves, Thermal and Conduction Properties of a PCL-biodegradable Gel Polymer Electrolyte with $\mathrm{LiClO}_{4}$, $\mathrm{LiF}_{3} \mathrm{CSO}_{3}$, and $\mathrm{LiBF}_{4}$ Salts, Int. J. Electrochem. Sci. 2 (2007) 52-63.

[23] Arup Dey, S. Karan, K.S. De, Effect of nanofillers on thermal and transport properties of potassium iodide-polyethylene oxide solid polymer electrolyte, Solid State Communications 149 (2009)1282-1287.

[24] H.J. Woo, S.R. Majid, A.K. Arof, Conduction and thermal properties of a proton conducting polymer electrolyte based on poly ( $\varepsilon$-caprolactone), Solid State Ionics 199-200 (2011)14-20.

[25] Zheng Shong, Qi Cao, Bo Jing, Xianyou Wang, Xiaoyun Li, Huayang Deng, Electrospun PVdF-PVC nanofibrous polymer electrolytes for polymer lithium-ion batteries, Mater. Sci. Eng. B 177 (2012) 86-91.

[26] M.M.E. Jacob, A.K. Arof, FTIR studies of DMF plasticized polyvinyledene fluoride based polymer electrolytes, Electrochimica Acta 45 (2000)1701-1706.

[27] D. Martin-Vosshage, B.V.R. Chowdari, Characterization of poly (ethylene oxide) with cobaltbromide, Solid State Ionics 62 (3-4) (1993) 205-216.

[28] Amrtha Bhide, Hariharan, K.; Ionic transport on $(\mathrm{PEO})_{6}: \mathrm{NaPO}_{3}$ polymer electrolyte plasticized with PEG400, Eur. Polym. J. 43 (2007) 4253-4270.

[29] Yogesh Kumar, Hashmi, S.A.; Pandey, G.P.; Ionic liquid mediated magnesium ion conduction in Poly (ethylene oxide) based polymer electrolyte, Electrochimica Acta 56 (2011) 3864-3873. 
[30] P. Dutta, S. Biswas, M. Ghosh, S.K. De, Chatterjee S, The dc and ac conductivity of polyaniline-polyvinly alcohol blends, Synth. Met. 122 (2) 2000 455-461.

[31] A.K. Johnscher, Analysis of the alternating current properties of ionic conductors, J. Mater. Sci. 13 (3) (1978) 553-562.

[32] M. Ravi, Y. Pavani, S. Bhavani, A.K. Sharma, V.V.R. Narasimha Rao, Investigations on Structural and Electrical Properties of $\mathrm{KClO}_{4}$ Complexed PVP Polymer Electrolyte Films, Int. J.Polym. Mater.Polym. Biomater. 61(5) (2012) 309-322.

[33] C. Leon, M.L. Lucia, J. Santamaria, Correlated ion hopping in single-crystal yttriumstabilized zirconia, Phys. Rev. B 55 (2) (1997) 882-887.

[34] K. Funke, Ion transport in fast ion conductors - spectra and models, Solid State Ionics 96 (1-4) (1997) 27-33.

[35] J.M. Le Meins, O. Bohnke, G. Courbion, Ionic conductivity of crystalline and amorphous $\mathrm{Na}_{3} \mathrm{Al}_{2}\left(\mathrm{PO}_{4}\right)_{2} \mathrm{~F}_{3}$, Solid State Ionics 111 (1998) 67-75.

[36] Y. Pavani, M. Ravi, S. Bhavani, A.K. Sharma, V.V.R. Narasimha Rao, Characterization of Poly(vinyl alcohol)/Potassium Chloride Polymer Electrolytes for Electrochemical Cell Applications, Polym. Eng. Sci. 52 (2012)1685-1692.

[37] G. Hirankumar, S. Selvasekarpandian, M.S. Bhuvaneswari, R. Baskaran, M. Vijayakumar $\mathrm{M}, \mathrm{Ag}^{+}$ion transport studies in a polyvinyl alcohol-based polymer electrolyte system, J. Solid State Electrochem. 10 (2006) 193-197.

[38] M.H. Khanmirzaei, S. Ramesh, Nanocomposite polymer electrolyte based on rice starch/ionic liquid/ $\mathrm{TiO}_{2}$ nanoparticles for solar cell application, Measurement 58 (2014) 68-72. 
[39] Agnieszka Swiderska-Mocek, Dominika Naparstek, Compatibility of polymer electrolyte base on N-methyl-N-propylpiperidinium bis (trifluoromethanesulphonyl) imide ionic liquid with $\mathrm{LiMn}_{2} \mathrm{O}_{4}$ cathod in Li-ion batteries. Solid State Ionics 26 (2014) 32-37.

[40] T. Tamilarasan, S. Ramaprabhu, Graphene based all-solid-state supercapacitors with ionic liquid incorporated polycrylonitrile electrolyte, Energy 5 (2013) 374-381.

[41] F.S. Howell, R.A. Bose, P.B. Macedo, C.T. Moynihan, Electrical relaxation in a glassforming molten salt, J. Phys. Chem. 78 (1974) 639-648.

[42] Silvia Gross, Daniele Camozzo, Vito Di Noto, Lidia Armelao, Eugenio Tondello, PMMA: A key macromolecular component for dielectric low-k hybrid inorganic-organic polymer films, Eur. Polym. J. 43 (2007) 673-696.

[43]S. Ramesh, M.F. Chai, Conductivity, dielectric behavior and FTIR studies of high molecular weight poly(vinylchloride)-lithium triflate polymer electrolytes, Mater. Sci. Eng. B139 (2007) 240-245.

[44] C. Leon, M.L. Lucia, J. Santamaria, M.A. Parsi, J. Sanz, A. Varez, Electrical conductivity relaxation and nuclear magnetic resonance of $\mathrm{Li}$ conducting $\mathrm{Li} 0.5 \mathrm{La} 0.5 \mathrm{TiO}_{3}$, Phys. Rev. B 54 (1) (1996)184.

[45] D. Teeters, R.G. Neuman, B.D. Tate, The concentration behavior of lithium triflate at the surface of polymer electrolyte materials, Solid State Ionics 85 (1996) 239-245.

[46] N. Kulshrestha, B. Chatterjee, P.N. Gupta, Characterization and electrical properties of polyvinyl alcohol based polymer electrolyte films doped with ammonium thiocyanate, Mater. Sci. Eng. B 184 (2014) 49-57. 
[47] R.J. Sengwa, S. Choudhary, S. Sankhla, Dielectric spectroscopy of hydrophilic polymersmontmorillonite clay nanocomposite aqueous colloidal suspension, Colloids Surface A: Physicochem. Eng. Aspects 336 (2009)79-87.

[48] M.C.R. Shastry, K.J. Rao, ac conductivity and dielectric relaxation studies in AgI-based fast ion conducting glasses, Solid State Ionics 44(3-4) (1991)187-198.

[49] D. Ravinder, A.V. Ramana Reddy, Ranga Mohan G, Abnormal dielectric behavior in polycrystalline zinc-substituted manganese ferrietes at high frequencies, Mater. Lett. 52(4-5) (2002) 259-265.

[50] S.L. Agrawal, M. Singh, M. Tripathi, M. Mauli Dwivedi, Kamlesh Pandey, Dielectric relaxation studies on [PEO-SiO 2 ]: $\mathrm{NH}_{4} \mathrm{SCN}$ nanocomposite polymer electrolyte films, J. Mater. Sci. 44 (2009) 6060-6068.

[51] J. Isasi, M.L. Lopez, M.L. Veiga, E. Ruiz- Hitzky, C. Pico, Structural Characterization and Electrical Properties of a Novel Defect Pyrochlore, J. Solid State Chem. 1995;116(2): 290.

[52] S. Ramesh, A.K. Arof, Ionic conductivity studies of plasticized poly(vinyl chloride) polymer electrolytes, Mater. Sci. Eng. B 85 (2001) 11-15.

[53] M.A.L. Nobre, S. Langfredi, Phase transition in sodium lithium niobate polycrystal:an overview based on impedance spectroscopy, J. Phys. Chem. Solids 62(2001)1999-2006. [54] K.P. Padmasree, D.K. Kanchan, Modulus studies of $\mathrm{CdI}_{2}-\mathrm{Ag}_{2} \mathrm{O}-\mathrm{V}_{2} \mathrm{O}_{5}-\mathrm{B}_{2} \mathrm{O}_{3}$ system Mater. Sci. Eng. B 122 (2005) 24-28.

[55] Archana Shukla, R.N.P. Choudhary, Impedance and modulus spectroscopy characterization of $\mathrm{La}^{+3} / \mathrm{Ma}^{+4}$ modified $\mathrm{PbTiO}_{3}$ nanoceramics, Curr. Appl. Phys. 11(3) (2011) 414-422. [56] A.K. Kulkarni, P. Lunkeneheimer, A. Loidl, Mixed alkali effect in the ac conductivity of glasses, Mater. Chem. Phys. 63 (1) (2000) 93-97. 
[57] H.J. Woo, S.R. Majid, A.K. Afor, Dielectric properties and morphology of polymer electrolytes based on poly ( $\varepsilon$-caprolactone) and ammonium thiocyanate, Mater. Chem. Phys. 134 (2012) 755-761.

[58] G.P. Pandey, S.A. Hashmi, Experimental investigations of an ionic-liquid-based, magnesium ion conducting, polymer gel electrolyte, J. Power Sources 187 (2009) 627-634. [59] A.K. Arof, M.Z, Kufian, M.F. Syukur, M.F. Aziz, A. E. Abdelrahman, S. R. Majid, Electrical double layer capacitor using poly(methyl methacrylate) $-\mathrm{C}_{4} \mathrm{BO}_{8} \mathrm{Li}$ gel polymer electrolyte and carbonaceous material from shells of mata kucing fruit, Electrochimica Acta 74 (2012) 39-45.

[60] S.A. Hashmi, Shunzo Suematsu, Katsuhiko Naoi, All solid-state redox supercapacitors based on supramolecular 1,5-diaminoanthraquinone oligomeric electrode and polymeric electrolytes, J. Power Sources 137 (2004) 145-151. 


\section{Captions for figures and tables}

Fig. 1. Macroscopic morphology of the pure PCL polymer film.

Fig. 2. DSC thermograms of PCL:LiSCN polymer electrolyte films with different LiSCN concentrations.

Fig. 3. Optical micrographs of pure PCL and PCL:LiSCN complexed polymer electrolyte films with different LiSCN contents: (a) pure PCL, (b) $5 \mathrm{wt} \% \mathrm{LiSCN}$, (c) $15 \mathrm{wt} \% \mathrm{LiSCN}$, and (d) 20 wt $\%$ LiSCN.

Fig. 4. Frequency-dependent ionic conductivities of the PCL:LiSCN (95:05) polymer electrolyte film at different temperatures. The inset shows the natural logarithm of DC conductivity $\times$ temperature $(\ln (\sigma T))$ as a functional of reciprocal temperature $(1 / T)$.

Fig. 5. Frequency-dependent conductivities of PCL:LiSCNpolymer electrolyte films with different LiSCN concentrations, measured at $303 \mathrm{~K}$.

Fig. 6. Frequency-dependent dielectric constants of the PCL:LiSCN (90:10) polymer electrolyte film at different temperatures.

Fig. 7. Frequency-dependent dielectric constants of PCL:LiSCN polymer electrolyte films with different LiSCN concentrations, measured at $303 \mathrm{~K}$.

Fig. 8. Frequency-dependent loss tangents for the PCL:LiSCN (95:05) complexed polymer electrolyte film at different temperatures.

Fig. 9. Temperature dependence of $\ln f_{\max }$ for the PCL:LiSCN (95:05) complexed polymer electrolyte film. $\ln (\sigma T)$ is also plotted for comparison.

Fig.10. Frequency dependences of $\tan \delta /(\tan \delta)_{\max }$ of PCL:LiSCN complexed polymer electrolytes films with different LiSCN concentrations, measured at $303 \mathrm{~K}$. 
Fig.11. Frequency-dependent $M^{\prime \prime}$ of the PCL:LiSCN (85:15) polymer electrolyte film at different temperatures.

Fig.12. Master electric modulus curves of the PCL:LiSCN (85:15) polymer electrolyte film at different temperatures.

Fig. 13. Cyclic voltammogram of the PCL:LiSCN (85:15) polymer electrolyte film with stainless steel electrodes, recorded at a scan rate $1 \mathrm{mV} \mathrm{s}^{-1}$, measured at $303 \mathrm{~K}$.

Table 1. Melting temperature $\left(T_{\mathrm{m}}\right)$, melting enthalpy $\left(\Delta H_{\mathrm{m}}\right)$ and relative crystallinity $\left(\chi_{\mathrm{c}}\right)$ of PCL:LiSCN complexed electrolyte films

Table 2. Conductivity $(\sigma)$, relaxation time $(\tau), n$ and $\beta$ values of PCL:LiSCN complexed electrolyte films 


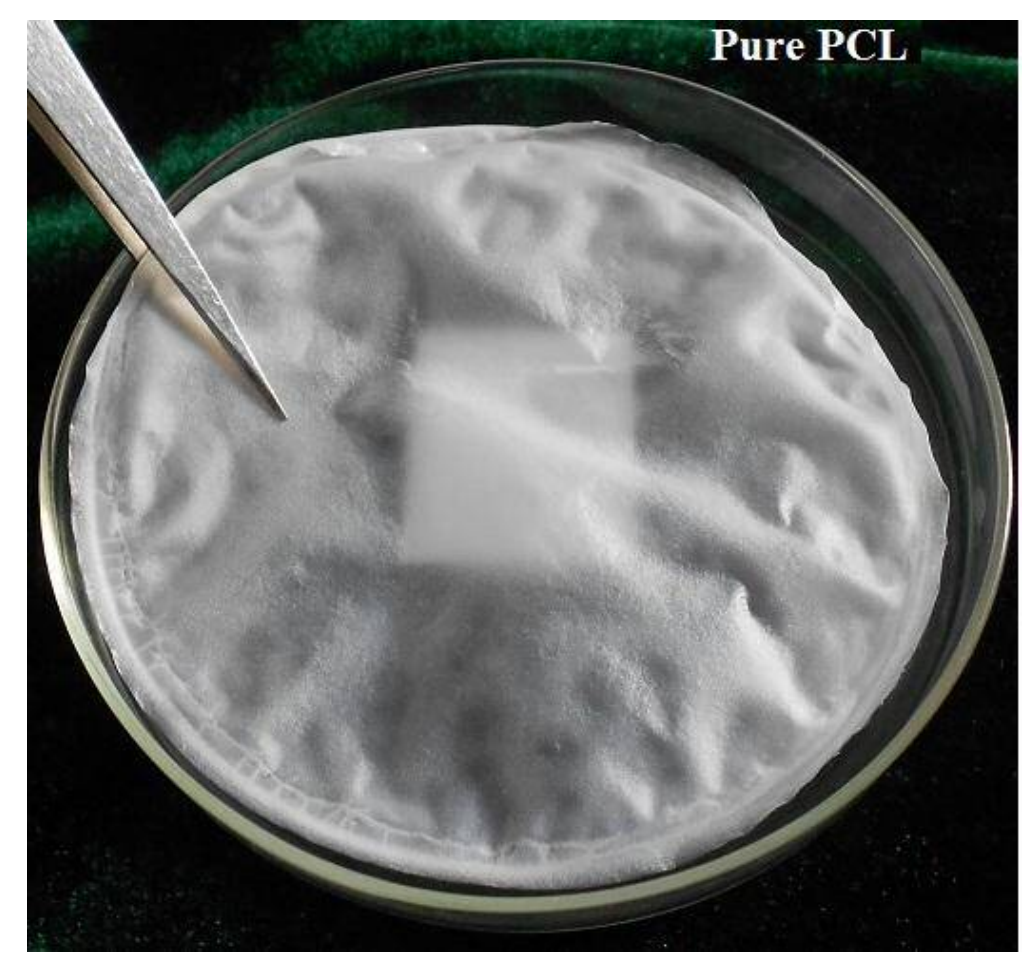

Fig. 1. Macroscopic morphology of the pure PCL polymer film.

.






Fig. 2. DSC thermograms of PCL:LiSCN polymer electrolyte films with different LiSCN concentrations. 

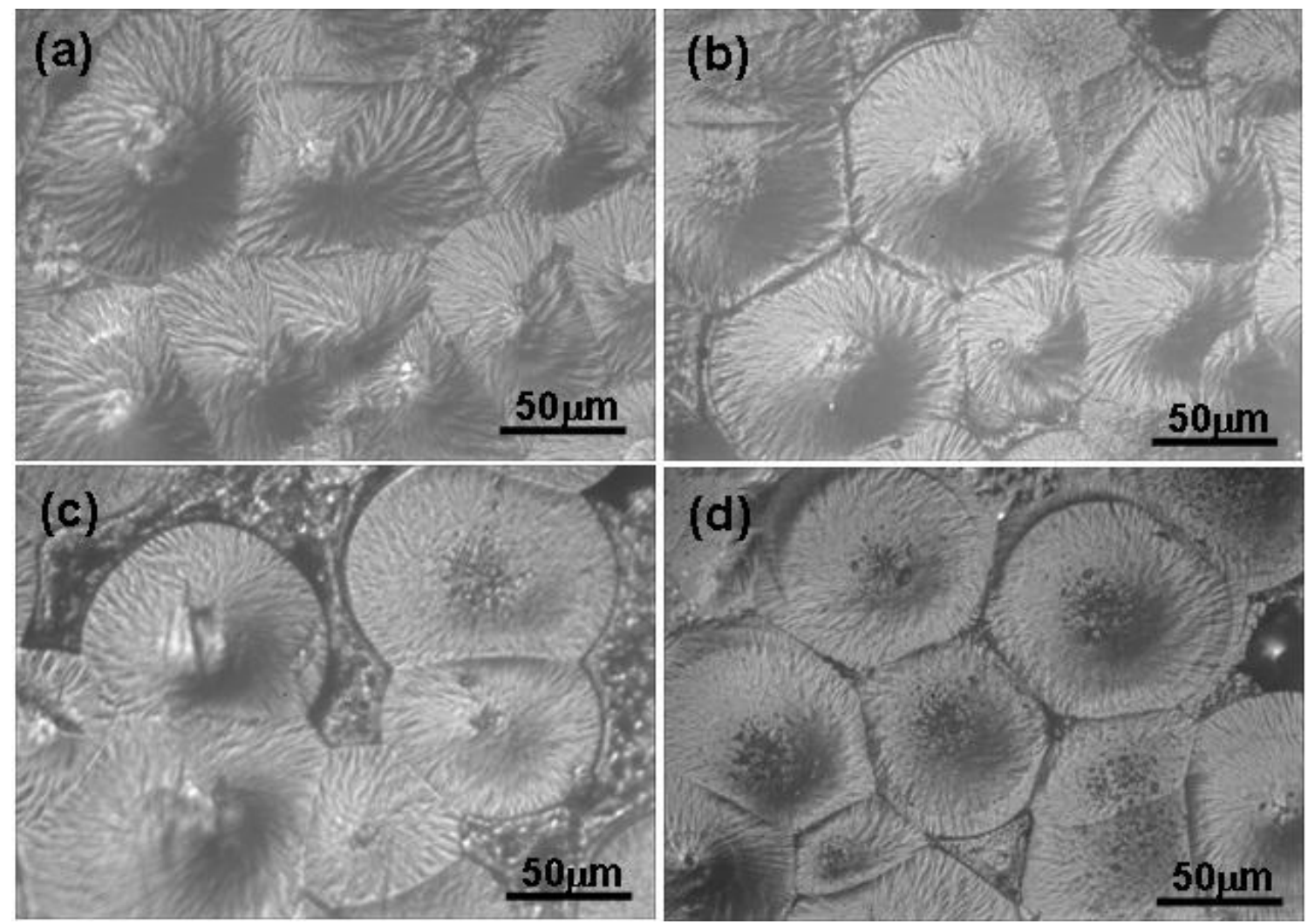

Fig. 3. Optical micrographs of pure PCL and PCL:LiSCN complexed polymer electrolyte films with different LiSCN contents: (a) pure PCL, (b) $5 \mathrm{wt} \% \mathrm{LiSCN}$, (c) $15 \mathrm{wt} \% \mathrm{LiSCN}$, and (d) 20 wt $\%$ LiSCN. 


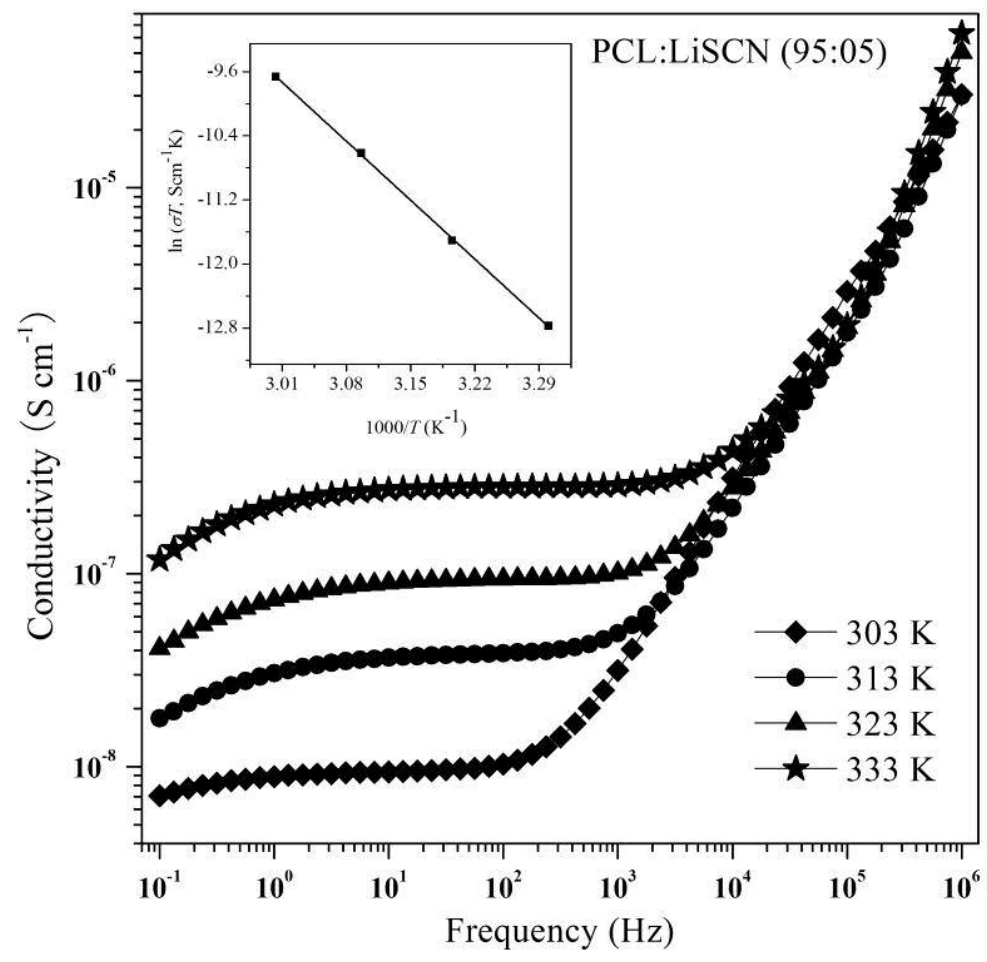

Fig. 4. Frequency-dependent ionic conductivities of the PCL:LiSCN (95:05) polymer electrolyte film at different temperatures. The inset shows the natural logarithm of DC conductivity $\times$ temperature $(\ln (\sigma T))$ as a functional of reciprocal temperature $(1 / T)$. 


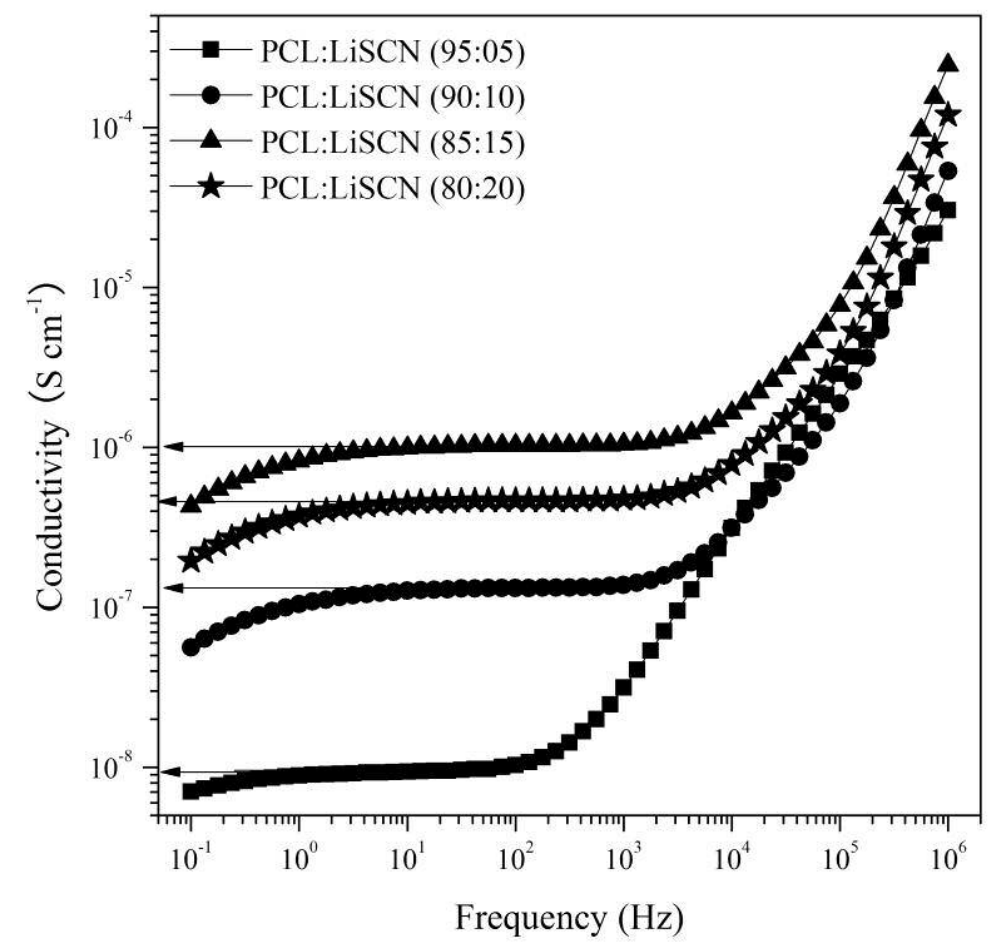

Fig. 5. Frequency-dependent conductivities of PCL:LiSCNpolymer electrolyte films with different LiSCN concentrations, measured at $303 \mathrm{~K}$. 




Fig. 6. Frequency-dependent dielectric constants of the PCL:LiSCN (90:10) polymer electrolyte film at different temperatures. 


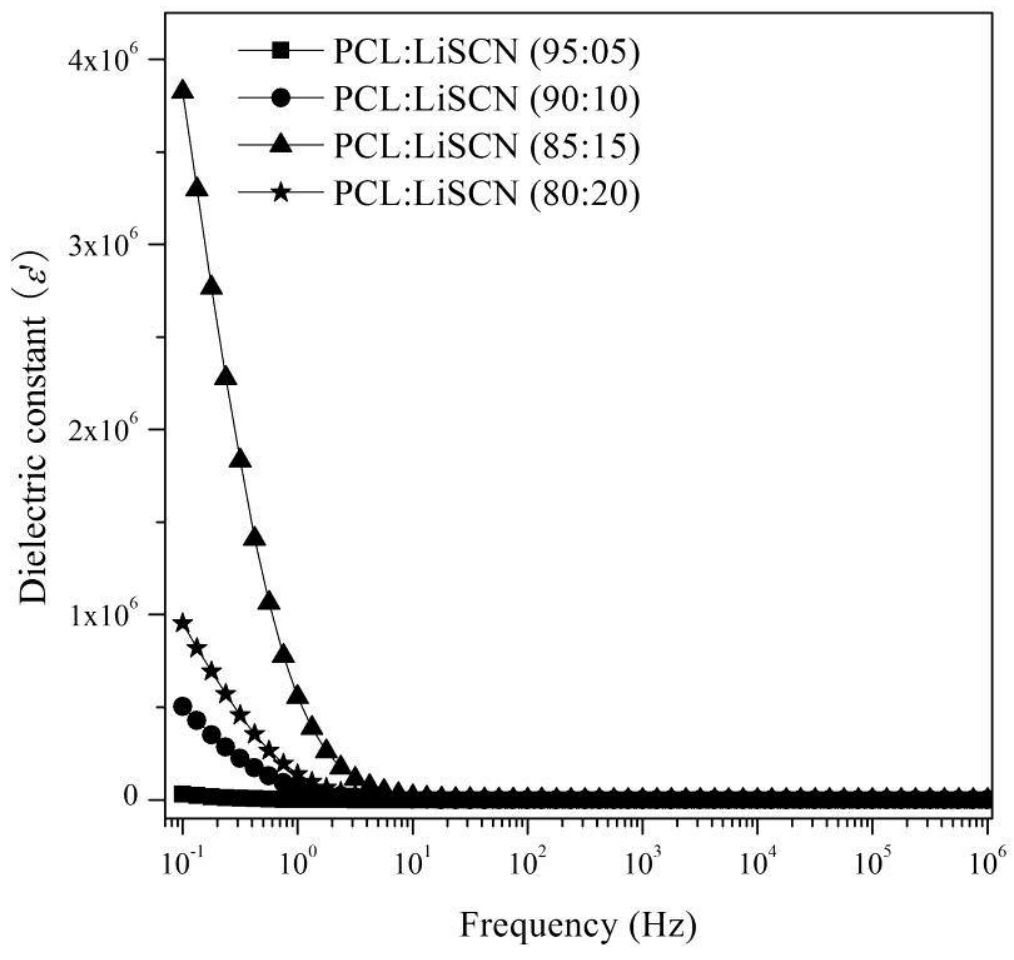

Fig. 7. Frequency-dependent dielectric constants of PCL:LiSCN polymer electrolyte films with different LiSCN concentrations, measured at $303 \mathrm{~K}$. 


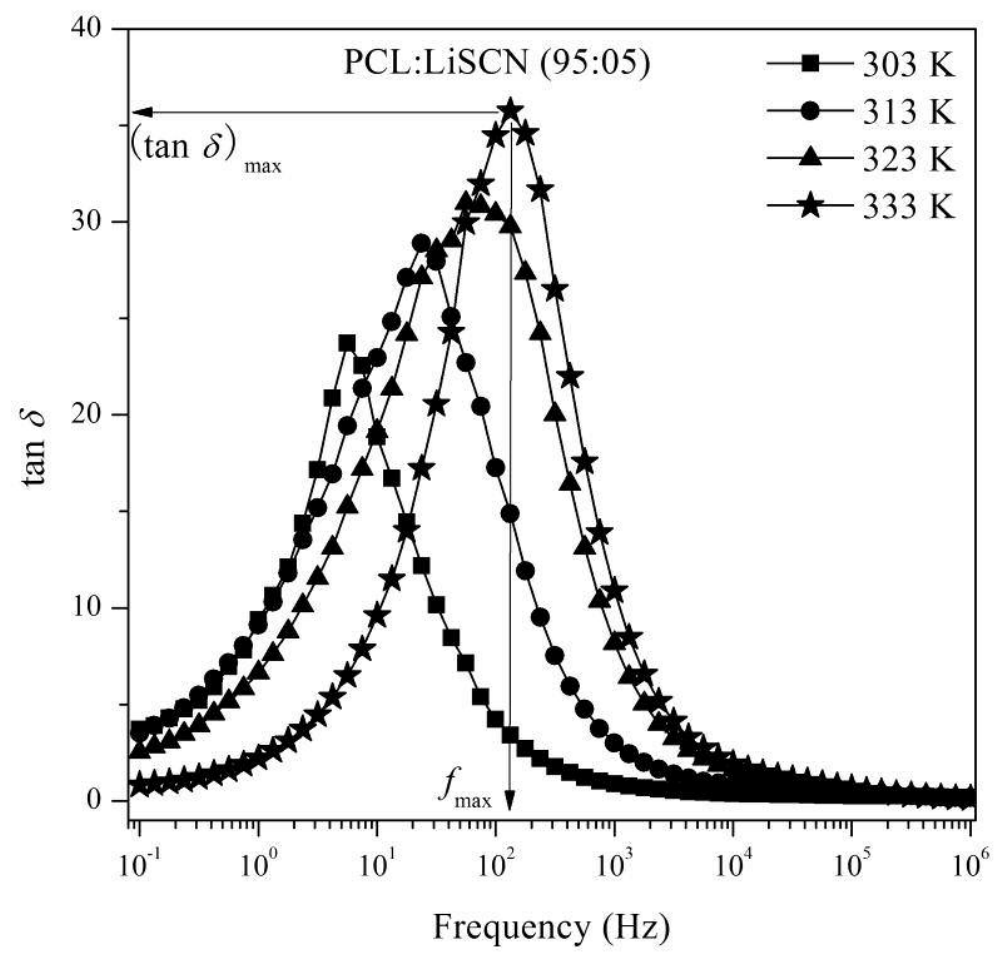

Fig. 8. Frequency-dependent loss tangents for the PCL:LiSCN (95:05) complexed polymer electrolyte film at different temperatures. 


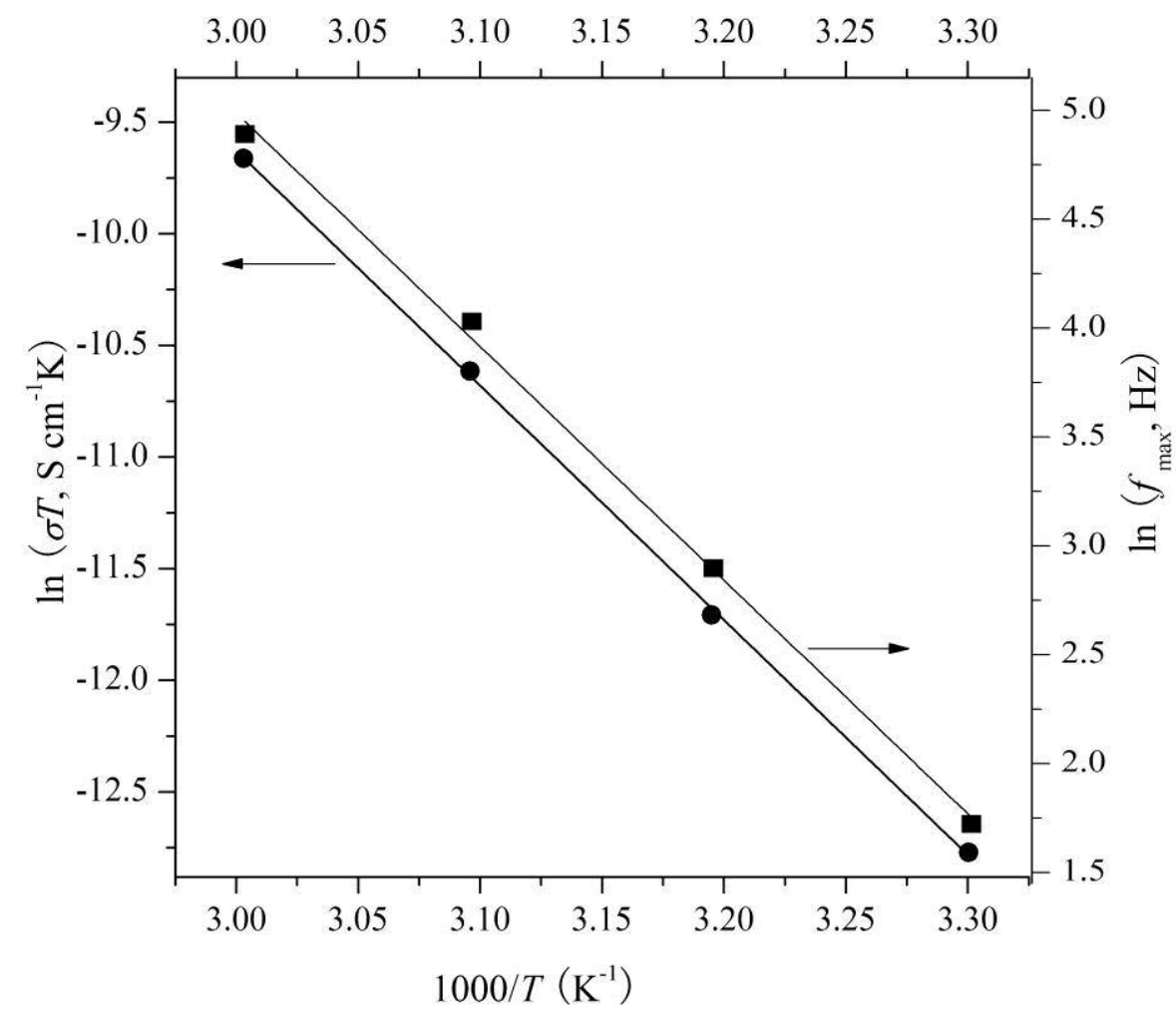

Fig. 9. Temperature dependence of $\ln f_{\max }$ for the PCL:LiSCN (95:05) complexed polymer electrolyte film. $\ln (\sigma T)$ is also plotted for comparison. 


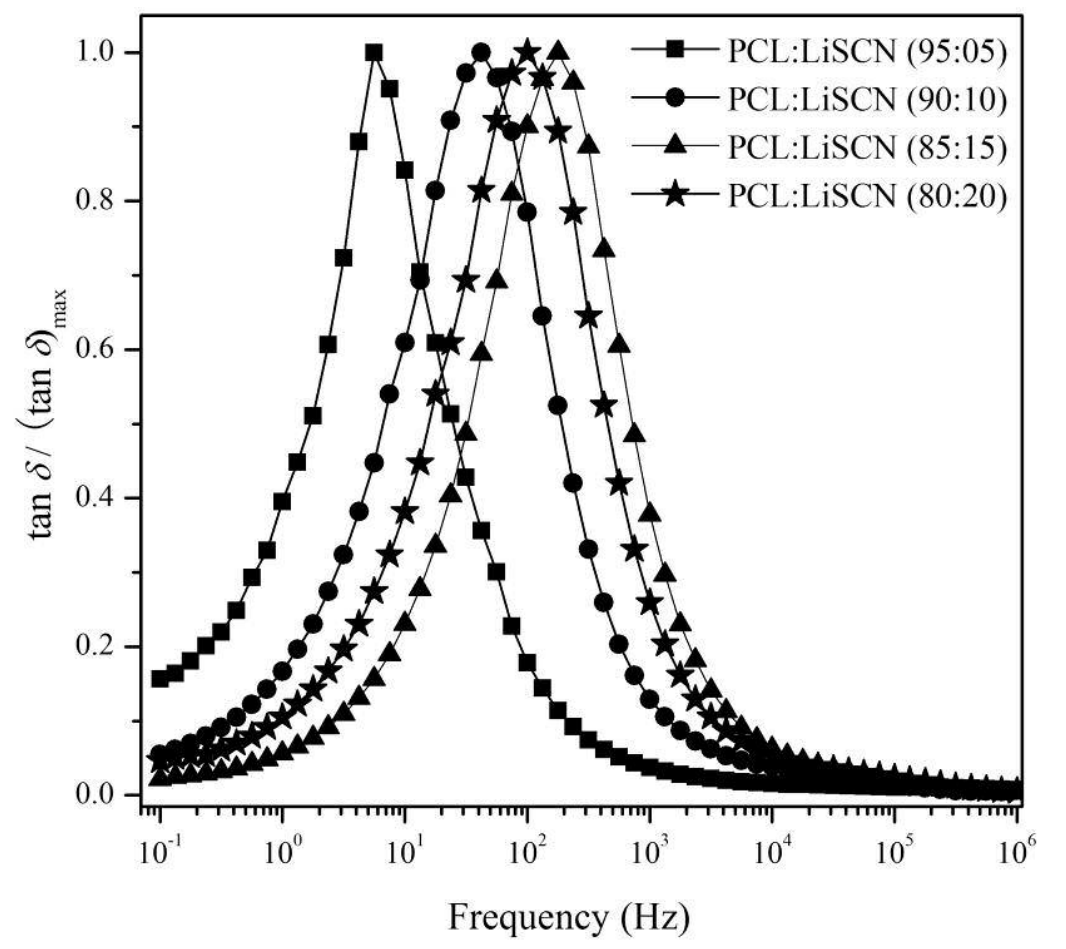

Fig.10. Frequency dependences of $\tan \delta /(\tan \delta)_{\max }$ of PCL:LiSCN complexed polymer electrolytes films with different LiSCN concentrations, measured at $303 \mathrm{~K}$. 


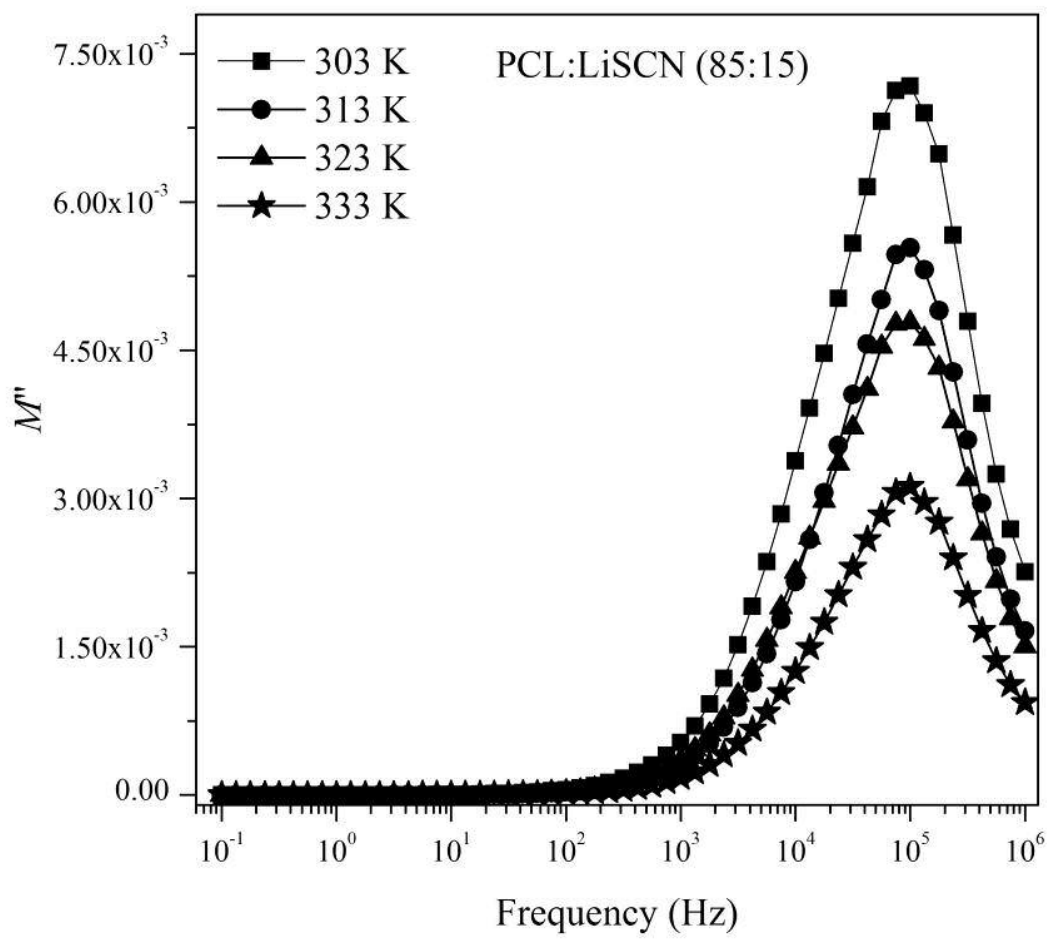

Fig.11. Frequency-dependent $M "$ of the PCL:LiSCN (85:15) polymer electrolyte film at different temperatures. 


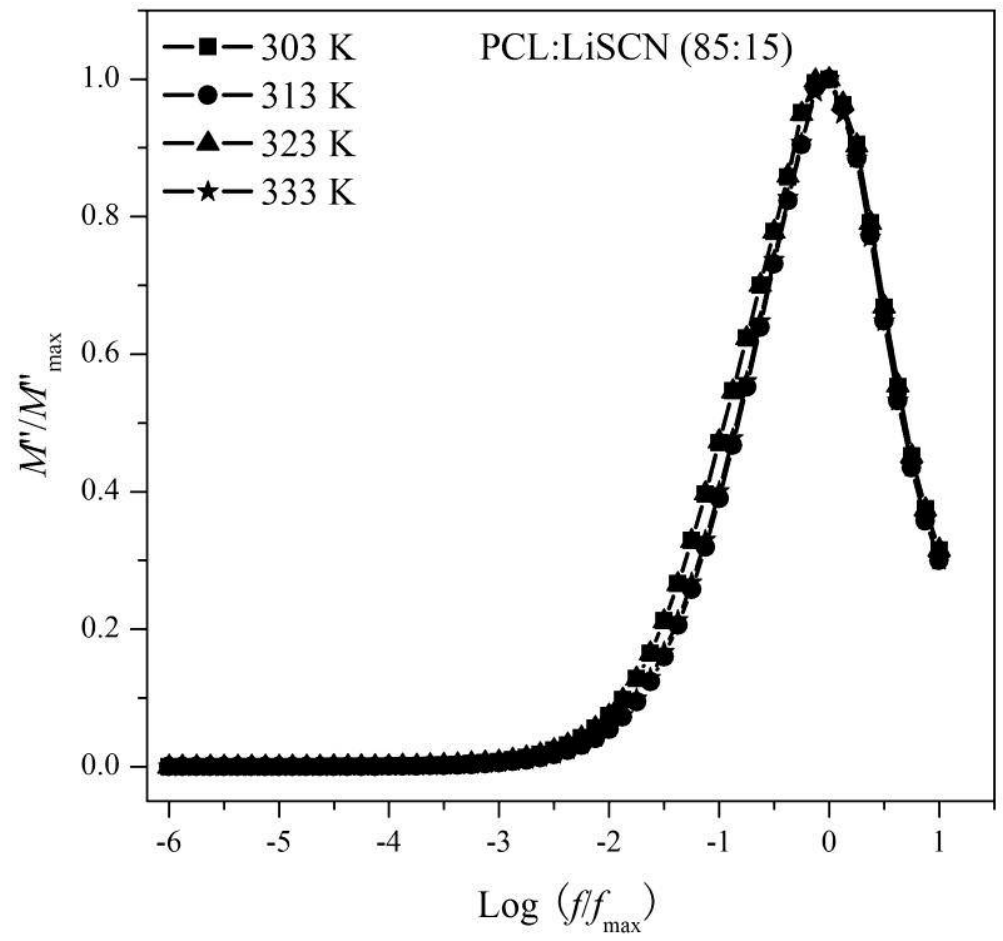

Fig.12. Master electric modulus curves of the PCL:LiSCN (85:15) polymer electrolyte film at different temperatures. 


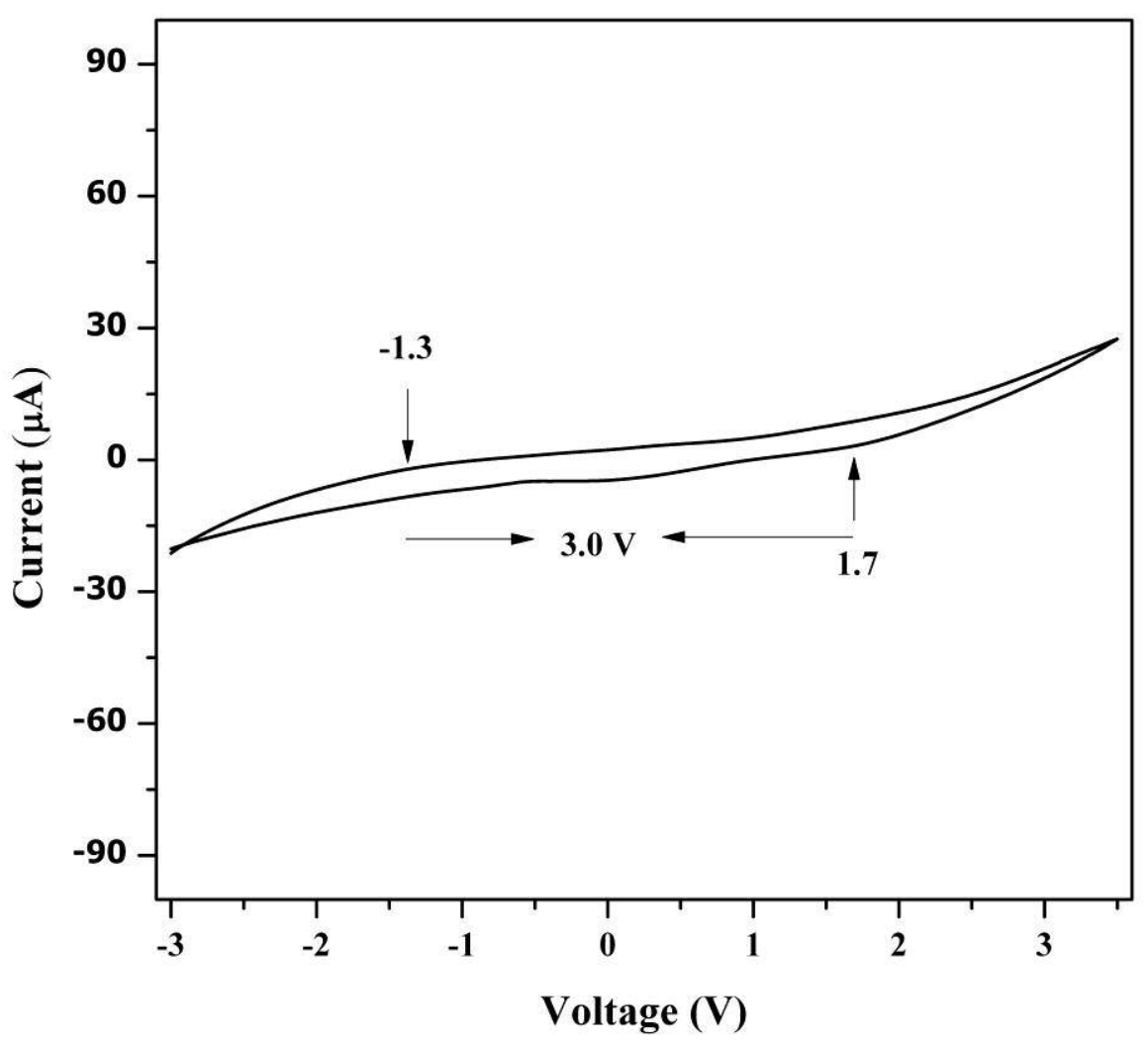

Fig. 13. Cyclic voltammogram of the PCL:LiSCN (85:15) polymer electrolyte film with stainless steel electrodes, recorded at a scan rate $1 \mathrm{mV} \mathrm{s}^{-1}$, measured at $303 \mathrm{~K}$. 
Table 1. Melting temperature $\left(T_{\mathrm{m}}\right)$, melting enthalpy $\left(\Delta H_{\mathrm{m}}\right)$ and relative crystallinity $\left(\chi_{\mathrm{c}}\right)$ of PCL:LiSCN complexed electrolyte films

\begin{tabular}{llll}
\hline Sample & $T_{\mathrm{m}}\left({ }^{\circ} \mathrm{C}\right)$ & $\Delta H_{\mathrm{m}}(\mathrm{J} / \mathrm{g})$ & $\chi_{\mathrm{c}}(\%)$ \\
\hline Pure PCL & 64.2 & 90.8 & 100 \\
PCL:LiSCN (95:05) & 61.8 & 82.7 & 90.0 \\
PCL:LiSCN(90:10) & 59.4 & 74.1 & 81.6 \\
PCL:LiSCN (85:15) & 57.8 & 66.7 & 73.4 \\
PCL:LiSCN(80:20) & 60.6 & 76.9 & 84.6 \\
\hline
\end{tabular}


Table 2. Conductivity $(\sigma)$, relaxation time $(\tau), n$ and $\beta$ values of PCL:LiSCN complexed electrolyte films

\begin{tabular}{lllll}
\hline Sample & $\sigma\left(\mathrm{S} \mathrm{cm}^{-1}\right)$ at $303 \mathrm{~K}$ & $\tau(\mathrm{s})$ & $n$ & $\beta$ \\
\hline PCL:LiSCN $(95: 05)$ & $9.36 \times 10^{-9}$ & $2.83 \times 10^{-2}$ & 1.20 & 0.736 \\
PCL:LiSCN (90:10) & $1.32 \times 10^{-7}$ & $3.77 \times 10^{-3}$ & 1.13 & 0.801 \\
PCL:LiSCN (85:15) & $0.10 \times 10^{-5}$ & $8.94 \times 10^{-4}$ & 0.96 & 0.933 \\
PCL:LiSCN (80:20) & $4.43 \times 10^{-7}$ & $1.59 \times 10^{-3}$ & 1.04 & 0.847 \\
\hline
\end{tabular}

